\title{
Pflege und Ethik. Aktuelle Herausforderungen
}

\author{
Helen Kohlen • Constanze Giese • Annette Riedel
}

Online publiziert: 1 . November 2019

(C) Springer-Verlag GmbH Deutschland, ein Teil von Springer Nature 2019

Eine gute Versorgung kranker und sterbender Menschen ist abhängig von einer guten Pflege. Gute Pflege ist abhängig von guten Rahmenbedingungen. Der aktuelle Pflegenotstand als erneuter und besonders schwerwiegender Mangel an Pflegefachkräften, verbunden mit ethisch relevanten Maßnahmen der Veränderung von Arbeitsbedingungen, diversen Maßnahmen der Personalrekrutierung oder auch die Definition von Mindestanforderungen an sprachliche und fachliche Kompetenzen der Pflegenden spiegeln sich im ethischen Diskurs, in den sozialethischen und gerechtigkeitsbezogenen Beiträgen wider. Unter den aktuellen Knappheitsbedingungen und den steigenden Anforderungen steht die Pflege folglich nicht nur vor politischen, sondern auch vor einer Vielzahl an ethischen Fragen. Wie kann beispielsweise ein pflegeethisch durchdachter ,guter“ Umgang mit neuen Technologien (u. a. Robotik) aussehen? Welche ethischen Kompetenzen sind aktuell und künftig in der Praxis erforderlich? Und was ist eine angemessene pflegeethische Position angesichts struktureller Mängel der aktuellen Pflege-Realität, die es wahrzunehmen gilt - jedoch ohne sich vor einer kritischen Pflegeperspektive zu scheuen, sondern vielmehr mit dem Ziel, alternative Entwicklungsmöglichkeiten in den Blick zu nehmen?

Univ. Prof. Dr. phil. H. Kohlen ( $\square)$

Philosophisch-Theologische Hochschule Vallendar (PTHV), Pallottistr. 3, 56179 Vallendar,

Deutschland

E-Mail: hkohlen@pthv.de

Prof. Dr. theol. C. Giese

Katholische Stiftungshochschule München, Preysingstraße 83, 81667 München, Deutschland

E-Mail: constanze.giese@ksh-m.de

Prof. Dr. phil. habil. A. Riedel, M.Sc.

Fakultät Soziale Arbeit, Gesundheit und Pflege, Hochschule Esslingen, University of Applied

Sciences, Flandernstr. 101, 73732 Esslingen, Deutschland

E-Mail: annette.riedel@hs-esslingen.de 
In Kürze: Welche Denk- und Lösungsansätze gibt es für die Entwicklung einer Pflegeethik in Wissenschaft und Praxis?

Die Entwicklung der Pflegeethik in Deutschland zeigt, dass theoretische Ansätze im Zusammenspiel mit der empirischen Sozialforschung und der praktischen Philosophie weiterentwickelt wurden. National haben ethische Fragen in der Pflege zwar in der Wissenschaft eher noch eine Randstellung, aber ihre Akteur*innen haben zunehmend pflegerische Themen in ethische Diskurse eingebunden und praxisrelevante Fragen auf die Agenda gerufen. Ergebnisse von Forschungsprojekten zu dezidiert pflegeethischen Fragestellungen liegen inzwischen vor. Die Platzierung der diversen pflegeethischen Forschungen in Theorie und Praxis in einem Themenheft „Ethik in der Pflege“ der Zeitschrift der Akademie für Ethik in der Medizin ist äußerst zeitgerecht, um den Stand der Forschung zu bündeln, den nationalen pflegeethischen Diskurs voranzubringen und auf geforderte Lösungsansätze zu reagieren.

Die Beschäftigung mit Themen der Pflegeethik - als einem eigenen Teilgebiet der Ethik im Bereich der Gesundheitsversorgung - zeigt sehr schnell spezifische thematische Schwerpunkte, die mit den derzeitigen Herausforderungen der pflegerischen Versorgung aufs Engste korrelieren. Die „Umstände“, unter denen sich pflegerisches Handeln als fürsorgliches Handeln realisieren soll, nehmen aktuell in der pflegeethischen Literatur eine besondere Rolle ein. Damit verbinden sich die spezifischen Perspektiven der Pflege auf professionsübergreifende Themen, wie die Frage der Beteiligung von Pflegenden an interprofessionellen, gemeinsamen ethischen Aufgaben wie der Ethikberatung und der ethischen Entscheidungsfindung. Interprofessionelle Zusammenarbeit auf Augenhöhe, wie sie in den diskursethischen Grundlagen aktueller Implementierungsformen der Ethikberatung - wie etwa Ethikkomitees notwendigerweise angelegt ist, muss oft erst noch hergestellt werden und kann nicht einfach nur postuliert werden. Dies ist um einer bestmöglichen Patientenversorgung willen immer wieder einzufordern. Dafür Lösungsmodelle zu entwickeln, kommt derzeit insbesondere der Pflegeethik zu. Dass solche Lösungsansätze dringend Not tun, um die Qualität der Pflege und die Attraktivität der Profession zu steigern, zeigt sich in umgekehrter Weise an der Rezeption des Themas ,,moral distress“, auf das die Pflege wie keine andere Profession im Gesundheitswesen ,angesprungen“ ist. Die Erfahrung, einer moralisch belastenden Situation tatsächlich oder auch nur scheinbar ohnmächtig gegenüber zu stehen, kumuliert in diesem Begriff. Er gibt Anlass zu einer vertieften Auseinandersetzung mit der ethischen Relevanz der Kooperationsbeziehungen der Heil- und Pflegeberufe genauso wie mit Fragen der faktischen ethischen Kompetenz der Akteur*innen. Empfundene Ohnmacht in hierarchischen Strukturen, in reproduzierten Sprachspielen und tradierten Berufsrollen wird durch anhaltendes Wissens- und Kompetenzgefälle auch im Bereich der Ethik perpetuiert. Dass der Ethikbildung in der pflegeethischen Literatur heute deutlich mehr Platz zukommt als in pflegepädagogischen Publikationen, erklärt sich vor diesem Hintergrund.

Das plakative „Wissen ist Macht“ lässt sich in der Pflegeethik transformieren in die Forderung adäquater Pflegebildungsmaßnahmen, welche die Akteur*innen erst befähigen und ermächtigen, gleichberechtigt mit den anderen Gesundheitsberufen gestaltend und verantwortlich in der Ethikarbeit, aber auch in der ethisch relevanten Standesvertretung zu kooperieren. 
Hinzu kommen die inhaltliche Diversität und Komplexität pflegethischer Fragen. Sie zeigen sich u.a. darin, dass in der Pflege- und Versorgungspraxis - aufgrund situativ divergierender moralischer und fachlicher Bewertungen - wiederholt Handlungs- und Entscheidungsunsicherheiten aufscheinen. In der Konsequenz bedeutet das, dass jede Entscheidung im Rahmen professioneller Pflege, d.h. auch in der Gesundheitsförderung und Prävention wie auch in der Begleitung am Lebensende, bewusst und verantwortungsvoll einem ethischen Abwägungs-, Reflexions- und Begründungsprozess zu unterziehen ist. Dies gilt insbesondere im Versorgungskontext vulnerabler Zielgruppen, die im pflegeberuflichen Handeln die Regel sind. Nicht nur in der dyadischen Struktur zwischen Pflegenden und Gepflegten, sondern in der Vielfalt aller Beteiligten und Betroffenen stellt sich die Frage nach Interessen, Intentionen und Zielen, nach (handlungs- und entscheidungs-)leitenden Werten und Direktiven. Hierbei geht es nicht nur um die jeweils individuell und situativ angemessene Entscheidung an sich, sondern auch um ein systematisches, verantwortungsvolles und kritisches Hinterfragen oder gar Unterbrechen von (bisherigen) Argumentationsmustern, von bisherigen Handlungs- und Entscheidungsautomatismen. Pflegende sind hierbei professionsbezogenen normativen Orientierungsdirektiven, Wertorientierungen, Leitlinien und Standards verpflichtet, welche eine ethisch verantwortbare, fachlich fundierte und individuell abgestimmte Versorgung absichern sollen. Die Komplexität pflegeberuflichen Handelns sowie der pflegespezifischen Situationen und Phänomene spiegeln nicht nur die erforderliche Perspektivenvielfalt, die notwendige (ethische) Sensibilität und die Bedeutsamkeit eines pflegeprofessionellen Standings, sondern verweisen auch auf die Notwendigkeit einer gefestigten pflegeberuflichen Expertise und Haltung, und in deren Folge auf eine umfassend fundierte und an aktuellen Entwicklungen und Erkenntnissen ausgerichtete Kultur der Aus-, Fort- und Weiterbildung. Die Beiträge des Heftes heben zugleich die Bedeutsamkeit der Pflege als eine zentrale Profession im Gesundheitswesen hervor, sie verdeutlichen den genuinen und situativen Beitrag professionell Pflegender zu ethisch gut begründeten Entscheidungen, und in der Konsequenz auch deren Beitrag zur Versorgungsqualität und zur Lebensqualität der zu pflegenden Menschen in unserer Gesellschaft.

Der erste Beitrag, von Monika Bobbert, enthält einen zentralen Appell: Bitte die ethische Perspektive nicht auf das moralische Handeln von Individuen und deren Interaktionen verengen, sondern den Blick sozialethisch erweitern! Kritisch und nah an aktuellen politischen Diskursen nimmt der Beitrag die ethischen Fragen der Pflege in Deutschland in den Blick. Die Autorin skizziert sechs sozialethische Probleme, deren Lösungen eine Voraussetzung für gerechtere Strukturen sind: (1) die Überwindung des Personalmangels; (2) der kritische Umgang mit Pflegebedarfsbeurteilungen; (3) die Reduktion bzw. Zurücknahme von Verdichtungsprozessen; (4) die Reduktion von Belastungen und Risikofaktoren für Pflegende; (5) die Bereitstellung von Ressourcen bei Belastungen; (6) die Gestaltung von Strukturen der Ethikberatung, die moralischen Stress verringern können. Bobbert argumentiert, dass die sozialethischen Fragen vorrangig vor den individualethischen Fragen zu behandeln seien, da der Blick auf die unmittelbar Betroffenen die Gefahr einer Problemverkürzung beinhalte. Letztlich seien oft dysfunktionale, gar ungerechte Strukturen die Ursache für ein Scheitern moralisch verantwortungsbewusster Individuen. 
Auch der zweite Beitrag von Constanze Giese beleuchtet kritisch und ebenso nah an gegenwärtigen gesellschaftspolitischen Diskursen die Bedingungen der (Un-)Gerechtigkeit für scheiternde Entwicklungen in der Pflege. Sie stützt sich auf die theoretischen Überlegungen zu den sozialen Praktiken der Unterdrückung von Iris Marion Young. Young hat darauf aufmerksam gemacht, dass formale Rechte und Beteiligungsmöglichkeiten für bestimmte Gruppen keine reale Entsprechung in der Praxis zur Folge haben und beispielsweise die Positionen von Frauen in bestimmten Entscheidungsfindungsprozessen nicht gleichberechtigt wirksam werden. Giese nutzt die von Young identifizierten fünf Formen der Unterdrückung (Ausbeutung, Marginalisierung, Machtlosigkeit, Kulturimperialismus, Gewalt) zur Analyse der pflegerischen Position und Rolle in der Gesellschaft mit besonderem Blick auf das Gesundheitssystem. Der Beitrag durchleuchtet die Mechanismen, die Pflege als reale Profession verhindern.

Der Beitrag von Helen Kohlen konturiert in einem ersten Schritt studienbasiert die aktuellen Herausforderungen, ethischen Bedenken und Fragen wie auch Beschränkungen und Unsicherheiten in der alltäglichen Pflegepraxis. Diese Klarlegung dient dem Nachvollzug der im zweiten Schritt ausgeführten Konsequenzen und Risiken (wie z.B. moralischer Stress, fehlende Beziehungen und Fragmentierung der Pflege) und ermöglicht es, den Zusammenhang zwischen aktuellen Kontextbedingungen und den unterschiedlichen Reaktionen und Widerständen der Pflegenden darauf zu erfassen. Die Perspektive verdeutlicht zugleich den Bedarf ethischer Beratung und Unterstützung durch die und mit den professionell Pflegenden. Die Frage, inwiefern und in welchem Umfang die Pflegenden in klinischen Ethikkomitees partizipieren und in welchem Ausmaß ihre Anliegen sowie die Stimmen der beteiligten Pflegenden gehört werden, wird anhand von Studienergebnissen der letzten drei Jahrzehnte aufgezeigt. Ergänzend wird die Perspektive auf sog. Pflegeethikkomitees gerichtet. Der Beitrag präzisiert die Diskrepanz zwischen dem Bedarf der Partizipation von Pflegenden in Strukturen institutioneller Ethikberatung und der Realität der eingeschränkten Partizipation sowie die fehlende Beachtung Pflegender und ihrer Anliegen in Ethikkomitees. Der Beitrag schließt mit konkreten Fragestellungen, die es ermöglichen, den Pflegenden in Ethikkomitees langfristig das dringend gebotene Gehör zu verschaffen und ihnen sowie ihren Anliegen Raum zu eröffnen. Hierbei wird der Bereitschaft zu demokratischen Lernprozessen eine gewichtige Rolle beigemessen.

Der Beitrag von Settimio Monteverde greift ein Phänomen auf, das in jüngster Zeit auch in der nationalen pflegeethischen Literatur zunehmend Beachtung und Eingang findet: der „moralische Stress“. Umso wichtiger ist die differenzierte Klarlegung und Kontextualisierung wie auch eine kritische Einordnung und definitorische Schärfung des Phänomens, um einer unreflektierten oder gar inflationären Nutzung des Begriffs „moralischer Stress“ entgegenzuwirken, aber auch um nachhaltige Interventionen im Sinne der professionell Pflegenden und der Pflegequalität generieren zu können. Der Beitrag bietet eine Protodefinition im Sinne einer modifizierten Begrifflichkeit, aus der sich eindeutige Konsequenzen für den Umgang mit moralischem Stress ableiten lassen. Die Differenzierung zwischen moralischem Dilemma, moralischem Stress und moralischer Ungewissheit verdeutlicht den Bedarf der vorliegenden Klarlegung in Bezug auf die jeweils angemessenen und passgenauen Interventionen. Der Beitrag 
bietet in der Folge eine Grundlage für die begründete und zielführende Auswahl von unterstützenden und entlastenden Maßnahmen in der Pflegepraxis.

Der Beitrag von Annette Riedel geht der Frage nach, wie die Entwicklung ethischer Kompetenz in der Pflege insbesondere in der hochschulischen Pflegebildung gemäß den Anforderungen des neuen Pflegeberufegesetzes angebahnt und gefördert werden kann. Da diese aktuelle gesetzliche Neuordnung die hochschulische Pflegeausbildung erstmals deutschlandweit als Regelfall einführt, stehen bislang hochschulspezifische, explizit methodische Vorschläge oder Empfehlungen zur Ethikbildung in Deutschland noch aus. Die Autorin argumentiert, dass neben der in der pflegeberuflichen Ausbildung üblichen Fallarbeit auch die Methode der Ethik-Leitlinienentwicklung als reflexionsbezogenes, reflexiv-diskursives Vorgehen einem alleinigen rezeptiven Aufnehmen relevanter Wissens- und Bildungsbestände im Kontext der Ethik überlegen sein kann. Damit lassen sich ethische Teilkompetenzen anbahnen und verdichten, hin zu einer pflegeprofessionellen Ethik-Expertise. Die Ethik-Leitlinienentwicklung kann Riedel zufolge den ethisch kompetenten Umgang mit ethischen Spannungsfeldern, die den pflegerischen Handlungs- und Verantwortungsbereich prägen, befördern, insbesondere dann, wenn die professionsethische Kompetenzentwicklung auch am Lernort Praxis flankiert wird.

Mit der Entwicklung ethischer Kompetenz am Lernort Praxis beschäftigen sich Sonja Lehmeyer und Annette Riedel. Sie fokussieren ausgehend von dem neuen Pflegeberufegesetz und seinen verbindlichen und veränderten Anforderungen an die Pflegebildung und Kompetenzentwicklung der künftigen Fachkräfte die besondere Bedeutung der Praxis als Lern- und Entwicklungsort auch pflegeethischer Kompetenzen. Die Rolle der Praxisanleiter*innen und die Anforderungen an deren ethische Kompetenzentwicklung werden in dem Bedingungsgefüge notwendiger Voraussetzungen und Rahmenbedingungen erörtert. Diese identifizieren die Autorinnen unter Bezugnahme auf Bildungsstrukturen und -prozesse zur Entwicklung der Ethikkompetenz (zukünftiger) Pflegefachkräfte. Resultierende Handlungsempfehlungen betreffen organisationsethische Aspekte wie die Etablierung von Strukturen der interprofessionellen ethischen Reflexion, der Stärkung der in der praktischen Berufsbildung tätigen Personen in ihrer Arbeit vor Ort, woraus sich Veränderungen der Rahmenbedingungen und Prozesse in den betreffenden Einrichtungen weit über die Anleitungssituation hinaus ergeben müssen.

Der abschließende Beitrag von Hartmut Remmers widmet sich den aktuellen ethischen Fragestellungen im Kontext von Pflege und Technik. Der zunehmende Einsatz von KI-Technologien und Assistenzsystemen im Kontext professioneller Versorgung fordert neben technischem Know-how insbesondere eine differenzierte ethische Auseinandersetzung. Hierbei geht es um den ethisch verantwortungsvollen Einsatz der Technik, dies insbesondere auch, um einen unreflektierten Einsatz zu verhindern. In dem Beitrag geht es ferner um die Fragen, wie sich die Pflege und deren Handlungsentscheidungen, wie sich die Pflege als genuine Beziehungsarbeit und wie sich die Patienten-Pflege-Beziehungen durch den Einsatz von Technik/von Assistenzsystemen verändern. Der Beitrag rückt Themen wie die Mensch-TechnikInteraktion in den Fokus und verweist auf die Ambiguitäten Pflegender in Bezug auf den Einsatz von Technik in der Pflege. Verschiedene Technikanwendungen werden analysiert und zu dem pflegeprofessionellen Auftrag und Handeln in Bezug 
gesetzt. Hierbei werden die jeweiligen Konsequenzen dargelegt und die Folgen für die Profession Pflege wie auch für das pflegeprofessionelle Handeln im Kontext der Technikanwendung kritisch und ethisch reflektiert. Die Auseinandersetzung mit Technikentwicklungen und deren ethische Bewertung sensibilisiert für aktuelle Fragen und mögliche (zukünftige) Kontroversen. Die Ausführungen eröffnen einen Diskurs, der eine Beteiligung von Pflegenden dringend erforderlich macht.

Danksagung Wir danken allen Autor*innen für ihre Beiträge und die vertrauensvolle Zusammenarbeit. Den externen Gutachter*innen danken wir für die hilfreichen Hinweise zu den Beiträgen. Wir danken der Schriftleitung von Ethik in der Medizin, dass dieses Themenheft ermöglicht wurde, und wir danken Thomas Schramme und Ralf J. Jox für die Begleitung auf dem Weg der Veröffentlichung sowie Andrea Kähling für ihre redaktionelle und organisatorische Arbeit.

Interessenkonflikt H. Kohlen, C. Giese und A. Riedel geben an, dass kein Interessenkonflikt besteht. 